\title{
Resiliencia de los docentes de la carrera de enfermería de la universidad técnica de Ambato ante la modalidad de teletrabajo
}

\author{
Diana Aracely Criollo Tituaña \\ dcriollo7266@uta.edu.ec \\ https://orcid.org/0000-0003-2326-0086
}

\author{
Mgtr. Eulalia Isabel Analuisa Jimenez \\ ei.analuisa@uta.edu.ec \\ https://orcid.org/0000-0002-7906-7640
}

Universidad Técnica de Ambato

Ambato - Ecuador

\section{RESUMEN}

A partir de la pandemia por COVID-19, la educación se trasladó a la modalidad virtual, instaurando el teletrabajo para los docentes, quienes asumieron nuevos retos relacionados al uso de la tecnología, lo que significo mayor inversión de tiempo y carga laboral. Por lo tanto, la presente investigación tuvo como objetivo determinar la resiliencia de los docentes de la Carrera de Enfermería de la Universidad Técnica de Ambato ante la modalidad de teletrabajo. En base a un enfoque cuantitativo, de tipo prospectivo, observacional, analítico de corte transversal, el cual contó con 74 participantes, a quienes se les aplicó un cuestionario basado en la escala de Resiliencia de Connor-Davinson (CDRISC) con un índice de alfa de Cronbach de 0.89. Entre los principales resultados se estableció que los docentes han sido resilientes ante la modalidad del teletrabajo, estableciendo que el 41,89\% de los docentes alcanza un nivel alto de resiliencia, mientras que el 40,54\% conservan un nivel medio. Por lo tanto, se concluye que tras las medidas adoptadas en la educación durante la pandemia por COVID-19, y la aplicación del teletrabajo, los docentes, han sido resilientes, desarrollando capacidades como la persistencia, la tenacidad, autoeficacia, adaptabilidad, control bajo presión, redes de apoyo y espiritualidad.

Palabras clave: resiliencia; teletrabajo; pandemia; covid-19; docentes. 


\title{
Resilience of the teachers of the career of nursing of the technical university of Ambato before the modality of teleworking
}

\begin{abstract}
Since the COVID-19 pandemic, education moved to the virtual modality, establishing telework for teachers, who took on new challenges related to the use of technology, which meant a greater investment of time and workload. Therefore, the objective of this research was to determine the resilience of the teachers of the Nursing Career of the Technical University of Ambato to the teleworking modality. Based on a quantitative, prospective, observational, analytical, cross-sectional approach, 74 participants were administered a questionnaire based on the Connor-Davinson Resilience Scale (CD-RISC) with a Cronbach's alpha index of 0.89. Among the main results, it was established that the teachers have been resilient to the teleworking modality, establishing that $41.89 \%$ of the teachers reached a high level of resilience, while $40.54 \%$ maintained a medium level. Therefore, it is concluded that after the measures adopted in education during the COVID19 pandemic, and the implementation of telework, teachers have been resilient, developing skills such as persistence, tenacity, self-efficacy, adaptability, control under pressure, support networks and spirituality.
\end{abstract}

Keywords: resilience, telework, pandemic, covid-19, teachers.

Artículo recibido: 30 noviembre. 2021 Aceptado para publicación: 29 diciembre 2021 Correspondencia: dcriollo7266@uta.edu.ec Conflictos de Interés: Ninguna que declarar 


\section{INTRODUCCIÓN}

A finales del 2019, en la ciudad de Wuhan en China, fue detectado por primera vez el virus del COVID-19, el cual pertenece a la familia de los coronavirus, mismo que en el ser humano ocasiona infecciones respiratorias, que parten desde un resfriado común hasta, enfermedades más graves, como el síndrome agudo severo (SARS-CoV2), qué se caracteriza por producir neumonía, dificultad respiratoria, sepsis y choque séptico. No obstante, el virus se propagó aceleradamente, alcanzando a otros territorios internacionales, de tal manera se convirtió en una problemática mundial, la cual el 11 de marzo del 2020 fue declarada como pandemia por parte de la Organización Mundial de la Salud (Ramón et al., 2020).

Tras la declaración de la pandemia, la Organización Mundial de la Salud pronunció un llamado a todos los países a tomar medidas para prevenir la propagación del virus y reducir al mínimo las consecuencias de la pandemia (OMS, 2020), las medidas tomadas en las naciones fueron críticas, ya que se experimentaron condiciones de confinamiento forzosos, distanciamiento social y paralización de actividades, lo cual provocó un cambio del estilo de vida de las personas (Ordorika y Ordorika, 2020).

Dentro del ámbito educativo, la pandemia por COVID-19, y las medidas que tomaron los países para su contención, afectaron con la suspensión masiva de las actividades presenciales de las instituciones educativas, lo que dio origen a un nuevo campo de acción a través de modalidades de aprendizaje a distancia, mediante la utilización de plataformas tecnológicas, que son utilizada en todos los niveles de formación (Marinoni et al., 2020). Es así que, en la educación superior, en todo el mundo al menos dos tercios de las instituciones lograron trasladar la enseñanza a la modalidad en línea, precisamente en América el $72 \%$ de las universidades lograron sustituir la enseñanza presencial por la enseñanza y aprendizaje a distancia (CEPAL, 2020).

La adopción acelerada de la modalidad virtual en la educación superior, orilló a instaurar el teletrabajo para los docentes, llevando a los maestros a asumir nuevos retos vinculados con el uso de las tecnologías de información y comunicación (TIC). Además, de acuerdo a la investigación realizada en México a los docentes de la Universidad Nacional Autónoma de México, se estableció que el teletrabajo, representa un incremento del 14\% de inversión de tiempo y carga laboral (Sánchez et al., 2020). Lo cual conlleva a que los profesores, tengan una alta probabilidad de sufrir consecuencias negativas en la salud 
(Grande de Prado et al., 2021).

En tal sentido, es necesario establecer mecanismos que faciliten la adaptación a los docentes a la modalidad de teletrabajo, conocido como resiliencia, misma que se define como la capacidad para afrontar exitosamente las adversidades, en la que se interrelacionan factores internos y externos para activar un proceso dinámico de adaptación positiva. Es decir, que en el campo de la docencia la resiliencia se enmarca en las respuestas o acciones de los profesores para adaptarse a las condiciones adversas de un entorno cambiante, especialmente durante la pandemia por COVID-19 (García del Castillo et al., 2016).

De acuerdo a estudios realizados sobre la resiliencia de docentes en distanciamiento social debido a la pandemia de distintos países de Iberoamérica como España, Argentina, Colombia, Ecuador, Portugal, República Dominicana, Uruguay, Paraguay, Portugal, Puerto Rico entre otros países, un total de 2.272 docentes, tras la aplicación de la escala de resiliencia de Connor Davison (CD-RISC 10), se obtuvo como resultado una asociación positiva entre los docentes con alta resiliencia y la percepción del evento disruptivo de la pandemia como una oportunidad, las habilidades socioemocionales para enfrentar los cambios, la experiencia de emociones de valencia positiva y la instrumentación de cambios en el estilo de vida durante la pandemia (Román et al., 2020). Dentro del Ecuador, en el estudio efectuado a los docentes de la facultad de pedagogía de la Universidad Técnica Luis Vargas Torres de la provincia de Esmeraldas, demuestra que la resiliencia de los docentes se halla en un nivel alto 70\%, y un nivel moderado en el $30 \%$, revelando la presencia de estabilidad emocional, que proyectó una mejor preparación para actuar de forma rápida y eficaz ante situaciones de amenaza. De la misma manera de acuerdo al género las docentes entre 51 y 60 años presentaron niveles de resiliencia altos. Indicando que las estrategias más utilizadas fueron focalizadas en la solución del problema, basadas en un plan de acción, y la reevaluación positiva de la situación para afrontar el estrés (Barahona, 2021).

Por consiguiente, es importante establecer la resiliencia que han desarrollado los docentes universitarios y las alternativas que han favorecido la pronta adaptación al teletrabajo y su reducción de probabilidad a sufrir consecuencias negativas. Es por ello que la presente investigación, desde un contexto local tiene como objetivo determinar la resiliencia de los docentes de la Carrera de Enfermería de la Universidad Técnica de Ambato ante la 
modalidad de teletrabajo.

De modo que la investigación es de alto impacto pues dentro del ámbito de la educación superior, enfatizando en el estudio de los docentes de la carrera de Enfermería, busca solventar con datos verídicos las experiencias y estrategias adaptadas para sobrellevar de forma eficiente los cambios en la educación online, sin que estos afecten de forma psicológica o influyan en la calidad de enseñanza y práctica de los docentes.

\section{ESTRATEGIAS METODOLÓGICAS O MATERIALES Y MÉTODOS}

La investigación se desarrolla bajo un enfoque cuantitativo, de tipo prospectivo, observacional, analítico de corte transversal, considerando como población objeto de estudio los docentes de la carrera de Enfermería de la Universidad Técnica de Ambato, siendo un total de 74 individuos, que corresponden a una población finita. Es así que se ha establecido una toma de datos censal.

Para la recolección de datos se utilizó un cuestionario basado en la escala de Resiliencia de Connor-Davinson (CD-RISC) que está formado por 25 ítems en la escala de Likert con cinco opciones de respuesta ("en absoluto", "rara vez", "a veces", "a menudo", y “casi siempre"), puntuadas de 0 (“en absoluto") a 4 (“casi siempre") (Broche et al., 2012). El instrumento se compone de cuatro factores: el primero aborda persistencia, tenacidad y autoeficacia (ítems 4,5,7,10,11,12,15,16,17,21,22,24,25); segundo factor adaptabilidad al cambio y control bajo presión (ítems 1,8,13,14,19), tercer factor asertividad y orientación positiva (ítems 6,18,20,23) y el factor redes de apoyo y espiritualidad (ítems 2,3). Cuenta además con un índice de alfa de Cronbach de 0.89 lo que valida el instrumento. Para el análisis del mismo las puntuaciones de cada ítem se suman y se interpreta que, a mayor puntuación en cada dimensión, más indicadores de resiliencia muestra el individuo, de tal modo que la puntuación máxima alcanzar es 100 (García et al., 2018).

La investigación guarda el derecho de confidencialidad de información, estipulada en los principios éticos de la Declaración de Helsinki, que se enfoca en la protección de la dignidad, autonomía, privacidad y confidencialidad de los datos de quienes participan en el estudio (Asamblea Médica Mundial, 2012), accediendo de forma voluntaria a proporcionar la información.

En la aplicación del instrumento de recolección se creó un formulario en línea conformado de dos secciones: la primera de datos sociodemográficos y la segunda del 
cuestionario de la escala de Resiliencia de Connor-Davinson (CD-RISC) mencionada anteriormente. Posterior a su diseño, se aplicó la encuesta a los docentes mediante Google Forms. Una vez recolectado los datos fueron analizados en el programa estadístico SPSS, para establecer hallazgos del estudio.

\section{RESULTADOS Y DISCUSIÓN}

Los docentes de la carrera de Enfermería de la Universidad Técnica de Ambato, que participaron en la investigación tienen en un 32,43\% entre 41 a 50 años y 51 a 60 años respectivamente, el 27,03\% entre 31 a 40 años y apenas el 8,11\% entre 20 a 30 años.

De acuerdo al género de los docentes de la carrera de Enfermería que participaron el $90,54 \%$ son mujeres y el 9,46\% hombres.

En relación al estado civil de los docentes participantes en la investigación el 58,11\% es casado/a, el 31,08\% soltero/a, 9,46\% divorciado/a y apenas el 1,35\% se halla en unión libre (Tabla 1).

Tabla 1. Datos sociodemográficos

\begin{tabular}{|l|l|c|c|}
\hline \multicolumn{2}{|l|}{ Ítem } & Recuento & $\%$ \\
\hline \multirow{5}{*}{ Edad } & Entre 20 - 30 años & 6 & $8,11 \%$ \\
\cline { 2 - 4 } & Entre 31-40 años & 20 & $27,03 \%$ \\
\cline { 2 - 4 } & Entre 41- 50 años & 24 & $32,43 \%$ \\
\cline { 2 - 4 } & Entre 51-60 años & 24 & $32,43 \%$ \\
\cline { 2 - 4 } & Total & 74 & $100,00 \%$ \\
\hline \multirow{4}{*}{ Género } & Mujer & 67 & $90,54 \%$ \\
\cline { 2 - 4 } & Hombre & 7 & $9,46 \%$ \\
\cline { 2 - 4 } & Total & 74 & $100,00 \%$ \\
\hline \multirow{4}{*}{ Estado civil } & Soltero/a & 23 & $31,08 \%$ \\
\cline { 2 - 4 } & Divorciado/a & 7 & $9,46 \%$ \\
\cline { 2 - 4 } & Casado/a & 1 & $58,11 \%$ \\
\cline { 2 - 4 } & Unión libre & 74 & $1,35 \%$ \\
\cline { 2 - 4 } & Total & 73 & $100,00 \%$ \\
\hline
\end{tabular}

Fuente: elaborado por Diana Criollo, en base a los datos recogidos de los docentes de la carrera de Enfermería.

En relación al análisis del factor uno que aborda el nivel de persistencia, tenacidad y autoeficacia, alcanza una puntuación media de 34,15 con una desviación de 17,55 en un rango mínimo de 0 y un máximo de 52. De igual manera, se obtuvo un valor t de 16, 74 con un nivel de significancia de 0,00 que es menor al valor $\mathrm{p}$ de 0,05 . De tal manera indica que los docentes de la carrera de Enfermería de la Universidad Técnica de Ambato si son 
persistentes, tenaces y autosuficientes (Tabla 2). Como lo sugiere el autor Vanderley et al. (2020) la persistencia, forma parte del concepto de resiliencia, ya que implica un proceso intersubjetivo desencadenado para lograr una respuesta a una situación de trauma. Relacionada con una conducta tenaz que se manifiesta por el control del pensamiento para encontrar soluciones a las adversidades (Erastro, 2020); es decir, conservar ideas positivas (Simbron, 2021). Por su parte la autoeficacia distingue un compromiso espiritual y temporal para la sostenibilidad en la vida y el entorno en que se desarrolla para socializar y la realización personal(Gordon, 2017).

Al analizar el factor dos relacionado a la adaptabilidad al cambio y control bajo presión, alcanzó una puntuación media del 13,16 con una desviación de 5,30, en una calificación mínima de 0 y máxima de 20, con un valor t de 21,38 y un nivel de significancia de 0,00 siendo inferior al valor $\mathrm{p}$ de 0,05 lo cual indica que los docentes de la carrera de Enfermería de la Universidad Técnica de Ambato si se adaptan al cambio y mantienen el control bajo presión (Tabla 2). Concordando con la noción de los autores Hernández y Cruz (2020) ya que sugieren que entre las habilidades para la acción dentro de la resiliencia, se establece un acelerado proceso de adaptación al cambio y control bajo presión. Dentro del ámbito educativo, el proceso de adaptación al cambio, cumple un proceso, que inicia con los retos y problemas que surgen por medio de la pandemia de COVID-19 y la nueva modalidad de trabajo que incurre, en un cambio de actitud, transformación tecnológica, pedagógica y socioemocional, que desencadena en un aprendizaje a nivel adaptativo. Así también el autor Carrillo García et al. (2018) establece que el control bajo presión, se caracteriza por un comportamiento en el que los trabajadores $\sin$ sacrificar la productividad adoptan condiciones que atenúa las modificaciones de carga laboral, toma de decisiones y control de recursos y habilidades para minimizar los efectos nocivos.

Tabla 2. Análisis del factor uno y dos

\begin{tabular}{|l|c|c|}
\hline Medidas & Factor uno & Factor dos \\
\hline Media & 34,15 & 13,16 \\
\hline Desv. Desviación & 17,55 & 5,30 \\
\hline Mínimo & 0,00 & 0,00 \\
\hline Máximo & 52,00 & 20,00 \\
\hline Valor t & 16,74 & 21,38 \\
\hline Nivel de significancia & 0,00 & 0,00 \\
\hline
\end{tabular}

Fuente: elaborado por Diana Criollo, en base a los datos recogidos de los docentes de la carrera de Enfermería. 
Por su parte, al estudiar el factor tres relacionado a la asertividad y orientación positiva, se obtuvo una puntuación media de 8,14 con una desviación de 3,55, en un rango mínimo de 0,00 y máximo de 16, alcanzando un valor t de 19,71 y un nivel de significancia de 0,00 inferior al valor p de 0,05 lo que indica que los docentes de la carrera de Enfermería de la Universidad Técnica de Ambato son asertivos y mantienen una orientación positiva ante la modalidad de teletrabajo (Tabla 3). De acuerdo con Pujol y Llacuna (2017) para fortalecer la resiliencia, la asertividad y orientación positiva se han desarrollado como parte del proceso adaptativo. Ya que el asertividad, permite expresar, sentimientos, emociones y pensamientos, liberando a la persona de la carga emocional negativa y mejora la comunicación (Naranjo, 2018).

Con respecto al cuarto factor de redes de apoyo y espiritualidad, se alcanzó una puntuación media de 4,91 con una desviación de 2,61 con un rango mínimo de 0,00 y máximo de 8,00, con un valor t de 16,15 y un nivel de significancia de 0,00 que es inferior al valor p de 0,05 lo que establece que los docentes de la carrera de Enfermería de la Universidad Técnica de Ambato conservan redes de apoyo y espiritualidad para ser resilientes al teletrabajo (Tabla 3). Es decir han generado respuestas amortiguadoras como, las redes de apoyo y espiritualidad, manifestadas en los docentes que han sido parte de la adaptación al teletrabajo, con el apoyo de capacitaciones para solventar las brechas existentes a la tecnología y la enseñanza, que ha fortalecido el dominio del teletrabajo a los docentes (UNICEF, 2020). Para ello, la espiritualidad le brinda al ser humano un sentido y un propósito que se materializa, dándole impulsos para enfrentar situaciones adversas (Irurzun y Yaccarini, 2018).

Tabla 3. Análisis del factor tres y cuatro

\begin{tabular}{|l|c|c|}
\hline \multicolumn{1}{|c|}{ Medidas } & Factor tres & Factor cuatro \\
\hline Media & 8,14 & 4,91 \\
\hline Desv. Desviación & 3,55 & 2,61 \\
\hline Mínimo & 0,00 & 0,00 \\
\hline Máximo & 16,00 & 8,00 \\
\hline Valor t & 19,71 & 16,15 \\
\hline Nivel de significancia & 0,00 & 0,00 \\
\hline
\end{tabular}

Fuente: elaborado por Diana Criollo, en base a los datos recogidos de los docentes de la carrera de Enfermería. 
Finalmente, la Resiliencia de Connor-Davinson (CD-RISC) alcanzó una puntuación media de 63,01, con un mínimo de 5 y un máximo de 96, alcanzando un valor t de 19,56 y un nivel de significancia del 0,00 que es menor al valor $\mathrm{p}$ de 0,05 lo que indica que los docentes de la carrera de Enfermería de la Universidad Técnica de Ambato han sido resilientes al teletrabajo instaurado durante la pandemia por COVID -19 (Tabla 4). Concordando con los autores Forján y Morelatto (2018) que establecen que la resiliencia en los docentes es una capacidad latente, dinámica y fluctuante, que depende de factores internos y externos, puesto que por vocación los docentes requieren de resiliencia emocional para manejar los desafíos y lograr enseñar al máximo pese a circunstancias de riesgo. De ahí que los docentes muestran ser resilientes ante la modalidad de teletrabajo, indicando que han sobrellevado situaciones de agotamiento y estrés a las que se enfrenta los maestros (Montero y Zuluaga, 2021).

Tabla 4. Análisis de Resiliencia

\begin{tabular}{|l|c|}
\hline Media & 63,01 \\
\hline Desv. Desviación & 27,71 \\
\hline Mínimo & 5,00 \\
\hline Máximo & 96,00 \\
\hline Valor t & 19,56 \\
\hline Nivel de significancia & 0,00 \\
\hline
\end{tabular}

Fuente: elaborado por Diana Criollo, en base a los datos recogidos de los docentes de la carrera de Enfermería.

De hecho, se ha establecido, que el 41,89\% de los docentes de la carrera de Enfermería de la Universidad Técnica de Ambato tienen un nivel alto de resiliencia, mientras que el $40,54 \%$ conservan un nivel medio y el 17,57\% alcanzan una resiliencia baja (Tabla 5). Como lo menciona el autor Pazos (2021) la conducta resiliente depende de la naturaleza de la situación y los factores personales, de tal manera el estímulo de estos factores favorecen el proceso de adaptación. Lo cual ha permitido que los docentes alcancen niveles altos de residencia. Para alcanzar tales niveles de resiliencia los docentes, han desarrollado cualidades como persistencia, tenacidad y autoeficacia. Que son, características particulares de una persona resiliente, tales como: fortalezas intrapsíquicas, habilidades para la acción, competencias de respuestas amortiguadoras (Franco, 2021). 
Tabla 5. Nivel de resiliencia.

\begin{tabular}{|c|c|c|}
\hline Niveles de estrés & Frecuencia & $\mathbf{\%}$ \\
\hline Baja & 13 & $17,57 \%$ \\
\hline Media & 30 & $40,54 \%$ \\
\hline Alta & 31 & $41,89 \%$ \\
\hline Total & 74 & $100 \%$ \\
\hline
\end{tabular}

Fuente: elaborado por Diana Criollo, en base a los datos recogidos de los docentes de la carrera de Enfermería.

Al establecer un análisis del nivel de resiliencia acorde a los datos sociodemográficos, de acuerdo a la edad se ha identificado que el $19 \%$ de las personas entre 41 a 50 años tiene una escala alta de resiliencia, en cuanto a los niveles medios el $16 \%$ de personas entre 51 a 60 años alcanza este estándar, las personas entre 31 años hasta los 50 años con un 5\% respectivamente tienen un nivel de resiliencia bajo (Tabla 6). Al mismo tiempo de acuerdo a la edad se ha identificado que las personas con una edad entre 20 a 30 años muestran los niveles más bajos de resiliencia. Estos resultados concuerdan con estudios previos, realizados a la población mexicana, en donde los jóvenes tienen menores niveles de resiliencia, pues de acuerdo con los autores González y Valdez (2013) establecen que la resiliencia es una capacidad que se desarrolla a través del ciclo de vida, por los factores internos y los dominios que se desarrollan por medio de la experiencia. De igual manera acorde al estudio efectuado por Delgado y Macias (2020) en los profesionales jóvenes entre 25 años de edad son más propenso a presentar cansancio emocional y despersonalización, consecuente con los años de experiencia pues quienes menos años de experiencia laboral tienen reportan niveles severos de agotamiento emocional, despersonalización y reducida realización. 
Tabla 6. Nivel de resiliencia según las características sociodemográficas

\begin{tabular}{|c|c|c|c|c|c|c|c|c|c|}
\hline & \multirow{2}{*}{ ítem } & \multicolumn{2}{|c|}{ Baja } & \multicolumn{2}{|c|}{ Media } & \multicolumn{2}{|c|}{ Alta } & \multicolumn{2}{|c|}{ Total } \\
\hline & & $\mathbf{f}$ & $\%$ & $\mathbf{F}$ & $\%$ & $\mathbf{f}$ & $\%$ & $\mathbf{f}$ & $\%$ \\
\hline \multirow{4}{*}{ Edad } & Entre 20 - 30 años & 2 & $3 \%$ & 2 & $3 \%$ & 2 & $3 \%$ & 6 & $8 \%$ \\
\hline & Entre 31 - 40 años & 4 & $5 \%$ & 10 & $14 \%$ & 6 & $8 \%$ & 20 & $27 \%$ \\
\hline & Entre 41- 50 años & 4 & $5 \%$ & 6 & $8 \%$ & 14 & $19 \%$ & 24 & $32 \%$ \\
\hline & Entre 51 - 60 años & 3 & $4 \%$ & 12 & $16 \%$ & 9 & $12 \%$ & 24 & $32 \%$ \\
\hline \multirow{2}{*}{ Género } & Mujer & 11 & $15 \%$ & 28 & $38 \%$ & 28 & $38 \%$ & 67 & $91 \%$ \\
\hline & Hombre & 2 & $3 \%$ & 2 & $3 \%$ & 3 & $4 \%$ & 7 & $9 \%$ \\
\hline \multirow{4}{*}{$\begin{array}{l}\text { Estado } \\
\text { civil }\end{array}$} & Soltero/a & 7 & $9 \%$ & 8 & $11 \%$ & 8 & $11 \%$ & 23 & $31 \%$ \\
\hline & Divorciado/a & 0 & $0 \%$ & 5 & $7 \%$ & 2 & $3 \%$ & 7 & $9 \%$ \\
\hline & Casado/a & 6 & $8 \%$ & 16 & $22 \%$ & 21 & $28 \%$ & 43 & $58 \%$ \\
\hline & Unión libre & 0 & $0 \%$ & 1 & $1 \%$ & 0 & $0 \%$ & 1 & $1 \%$ \\
\hline
\end{tabular}

Fuente: elaborado por Diana Criollo, en base a los datos recogidos de los docentes de la carrera de Enfermería.

De acuerdo al género el $38 \%$ de las mujeres tiene un nivel alto de resiliencia mientras que los hombres apenas alcanzan un $4 \%$, por su parte el $38 \%$ de las mujeres conserva niveles moderados al igual que el 3\% de hombres, además en las mujeres en un 15\% tienen niveles bajos de resiliencia al igual que el 3\% de los hombres (Tabla 6). Tales resultados concuerdan con la noción del autor Ramirez (2021), quien indica que las mujeres tienen una capacidad desarrollada para afrontar retos y adversidades, tanto en la vida personal, familiar, pareja, trabajo, ya que éstas requieren de la toma de decisiones acertadas con mayor flexibilidad de respuestas, que son innatos de las mujeres pues desde su tolerancia a los cambios físicos en cada etapa del ciclo de vida, a la lucha constante por la igualdad de género, han posicionado a la mujer como un símbolo de resiliencia. De igual forma según el estado civil el $28 \%$ de los casados/as tiene una resiliencia alta, y el $22 \%$ de casados una resiliencia media, mientras que el $9 \%$ de solteros conserva niveles bajos de resiliencia (Tabla 6).

\section{CONCLUSIÓN O CONSIDERACIONES FINALES}

Los docentes de la carrera de Enfermería de la Universidad Técnica de Ambato, han mostrado ser resilientes ante la modalidad de teletrabajo, adoptada como medida para continuar con la educación durante la pandemia de COVID-19, ya que se establece que 
los docentes universitarios alcanzan en un 41,89\% niveles altos de resiliencia. Lo que es un indicador alentador ya que demuestra la capacidad de respuesta acertada por parte de los profesionales ante el teletrabajo.

Tras la aplicación del cuestionario basado en la escala de Resiliencia de Connor-Davinson (CD-RISC), se ha identificado que el factor predominante en los docentes de la carrera de Enfermería de la Universidad Técnica de Ambato ha sido el factor uno con una media de 34,15 , compuesto por las dimensiones de persistencia, tenacidad y autoeficacia. Lo que les ha permitido tomar decisiones y encontrar soluciones ante los problemas eventuales del teletrabajo, con un latente compromiso hacia la enseñanza de calidad.

De acuerdo a los factores sociodemográficos de la población estudiada, con relación a la edad se ha establecido que los docentes entre 41 años y 50 años muestran niveles altos de resiliencia, debido a que mientras mayor experiencia tenga es menos propenso a sufrir condiciones severas de agotamiento emocional, despersonalización y reducida realización en el ámbito profesional. Por su parte el género predominante en la investigación ha sido el femenino de tal manera, es quien conserva los mayores índices de resiliencia.

Finalmente, para conservar niveles altos de resiliencia en los docentes, se requiere más halla de desarrollar cualidades internas como: tenacidad, perseverancia, control bajo presión y auto eficiencia, es necesario contar con la ayuda externa, que parte desde la capacitación constante para sobrepasar las brechas tecnológicas en la enseñanza, de igual manera el fortalecimiento de los lazos y colaboración entre compañeros de trabajo y la comunicación acertada que permite la externalización de emociones para la solución de posibles conflictos.

\section{LISTA DE REFERENCIAS}

Asamblea Médica Mundial, H. (2012). Declaración de Helsinki de la AMM - Principios éticos para las investigaciones médicas en seres humanos. (Vol. 66). http://repositorio.mederi.com.co/bitstream/handle/123456789/386/DeclaracionHelsinki-2013-Esp.pdf? sequence $=1$

Barahona, S. (2021). Resiliencia y estrategias de afrontamiento del estrés laboral durante la pandemia del covid-19 en los docentes de la facultad de la pedagogía de la Universidad Técnica Luis Vargas Torres de Esmeraldas. Repositorio Pontificia Universidad Católica Del Ecuador. 
https://repositorio.pucese.edu.ec/bitstream/123456789/2536/1/Mina Barahona Sonia Malia.pdf

Broche-Pérez, Y., Rodríguez-Martín, B. C., Pérez Santaella, S., Alonso Díaz, G., Hernández Carballo, A., \& Blanco, Y. (2012). Boris C . Rodríguez Martín y Osana Molerio Pérez. Feijoo, 1(October).

Carrillo García, C., Ríos Rísquez, M. I., Escudero Fernández, L., \& Martínez Roche, M. E. (2018). Factores de estrés laboral en el personal de enfermería hospitalario del equipo volante según el modelo de demanda-control-apoyo. Enfermeria Global, 17(2), 315-324. https://doi.org/10.6018/eglobal.17.2.277251

CEPAL. (2020). La educación en tiempos de la pandemia de COVID-19. Cepal. https://www.cepal.org/es/publicaciones/45904-la-educacion-tiempos-lapandemia-covid-19

DELGADO, B. T. B., \& MACIAS, H. C. K. (2020). Estrés Laboral Del Personal Médico Durante La Pandemia Covid 19 Hospital General Monte Sinaí. www.fcm.ug.edu.ec

Erastro, E. D. (2020). Los Sorprendentes Resultados Del Pensamiento Tenaz. https://es.scribd.com/doc/305107060/Los-Sorprendentes-Resultados-DelPensamiento-Tenaz

Forján, R., \& Morelatto, G. (2018). Estudio comparativo de factores de resiliencia en docentes de contextos socialmente vulnerables. PSICOGENTE, 21(40). https://doi.org/10.17081/psico.21.40.3075

Franco, D. (2021). Vista de Directivos docentes resilientes. Revista Oratores. https://revistas.umecit.edu.pa/index.php/oratores/article/view/413/840

García del Castillo, J. A., García del Castillo-López, Á., López-Sánchez, C., \& Dias, P. C. (2016). Conceptualización teórica de la resiliencia psicosocial y su relación con la salud. Health and Addictions/Salud y Drogas, 16(1), 59-68. https://doi.org/10.21134/haaj.v16i1.263

García León, M. Á., González-Gomez, A., Robles-Ortega, H., Padilla, J. L., \& PeraltaRamirez, I. (2018). Propiedades psicométricas de la Escala de Resiliencia de Connor y Davidson (CD-RISC) en población española. Anales de Psicología, 35(1), 33-40. https://doi.org/10.6018/analesps.35.1.314111

González-Arratia López Fuentes, N. I., \& Valdez Medina, J. L. (2013). Resiliencia: 
Diferencias por Edad en Hombres y Mujeres Mexicanos. Acta de Investigación Psicológica, 3(1), 941-955. https://doi.org/10.1016/S2007-4719(13)70944-X

Gordon, B. H. (2017). Iniciativa de autosuficiencia.

https://www.churchofjesuschrist.org/bc/content/ldsorg/topics/self-reliance/Self-

Reliance_Training_002_FINAL_PDF_NO NOTES.pdf

Grande de Prado, M., García Peñalvo, F. J., Corell Almuzara, A., \& Abella García, V. (2021). Evaluación en Educación Superior durante la pandemia de la COVID-19. Campus Virtuales : Revista Científica Iberoamericana de Tecnología Educativa, 10(1), 2021. www.revistacampusvirtuales.es

Hernández, P., \& Cruz, F. (2020). Vista de Adaptación al cambio en tiempos de pandemia el caso de una Universidad Pública en México. Gestión y Estrategia. http://gestionyestrategia.azc.uam.mx/index.php/rge/article/view/683/606

Irurzun, J. I., \& Yaccarini, C. (2018). Resiliencia, Espiritualidad y Propósito de Vida. Una revisión del estado del arte. Psocial, 4(2), 58-66. https://www.researchgate.net/publication/330076139_Resiliencia_Espiritualidad _y_Proposito_de_Vida_Una_revision_del_estado_del_arte

Marinoni, G., Van't Land, H., \& Jensen, T. (2020). The impact of Covid-19 on higher education around the world.IAU Global Survey Report. In Iau-Aiu.Net. https://www.youtube.com/channel/UCT5nt5FGVklxrtUHinF_LFA

Montero, K., \& Zuluaga, S. (2021). Resiliencia y Estrés Laboral en los instructores del Servicio Nacional de Aprendizaje. Universidad de La Costa. https://repositorio.cuc.edu.co/bitstream/handle/11323/8809/Resiliencia y Estrés Laboral en los instructores del Servicio Nacional de Aprendizaje\%2C SENA.pdf? sequence $=1 \&$ is Allowed=y

Naranjo Pereira, M. L. (2018). El autoconcepto positivo; un objetivo de la orientación y la educación. Actualidades Investigativas En Educación, 6(1). https://doi.org/10.15517/AIE.V6I1.9192

OMS, O. M. de la S. (2020). Cronología de la respuesta de la OMS a la COVID-19. World Health Organization Covid 19. https://www.who.int/es/news/item/29-062020-covidtimeline

Ordorika, I., \& Ordorika, I. (2020). Pandemia y educación superior. Revista de La Educación Superior, 49(194), $1-8$. 
https://doi.org/10.36857/RESU.2020.194.1120

Pazos Solari, J. G. (2021). Relación entre resiliencia y estrés en personal de la salud. In Repositorio Institucional

Ulima. https://repositorio.ulima.edu.pe/handle/20.500.12724/13229

Pujol Franco, L., \& Llacuna Morera, J. (2017). NTP 667 : La conducta asertiva como habilidad social. Ministerio de Trabajo y Asuntos Sociales España, 5. https://www.redalyc.org/pdf/870/87041161004.pdf

Ramirez, E. (2021). La resiliencia: El arma secreta de la mujer. Neuro Resiliencia. https://www.neuroresiliencia.com/la-resiliencia-el-arma-secreta-de-la-mujer/

Ramón, M., Abreu, P., Jesús Gómez Tejeda, J., Alejandro, R., \& Guach, D. (2020). Características clínico-epidemiológicas de la COVID-19. Revista Habanera de Ciencias

Médicas. http://www.revhabanera.sld.cu/index.php/rhab/article/view/3254/2505

Román, F., Forés, A., Calandri, I., Gautreaux, R., Antúnez, A., Ordehi, D., Calle, L., Poenitz, V., Correa Pérez, K. L., Torresi, S., Barcelo, E., Conejo, M., Ponnet, V., \& Allegri, R. (2020). Resiliencia de docentes en distanciamiento social preventivo obligatorio durante la pandemia de COVID-19. Journal of Neuroeducation, 1(1), 76-87. https://doi.org/10.1344/joned.v1i1.31727

Sánchez Mendiola, M., Martínez Hernández, A. M. del P., Torres Carrasco, R., de Agüero Servín, M., Hernández Romo, A. K., Benavides Lara, M. A., Rendón González, V. J., \& Jaimes Vergara, C. A. (2020). Retos educativos durante la pandemia de COVID-19: una encuesta a profesores de la UNAM. Revista Digital Universitaria, 21(3). https://doi.org/10.22201/codeic.16076079e.2020.v21n3.a12

Simbron, N. (2021). ¿Qué es la tenacidad en psicologia? https://aleph.org.mx/que-es-latenacidad-en-psicologia

UNICEF. (2020). Consejos para docentes. In Unicef Mexico. https://www.unicef.org/mexico/consejos-para-docentes

Vanderley, I. C. S., Vanderley, M. de A. S., Santana, A. D. da S., Scorsolini-Comin, F., Neto, W. B., \& Monteiro, E. M. L. M. (2020). Factores relacionados con la resiliencia de adolescentes en contextos de vulnerabilidad social: revisión integradora. In Enfermeria Global (Vol. 19, Issue 3, pp. 582-625). Universidad de Murcia. https://doi.org/10.6018/eglobal.411311 